\title{
Precise Endoscopic and Pathologic Features in a Crohn's Disease Case with Two Fistula-associated Small Bowel Adenocarcinomas Complicated by an Anal Canal Adenocarcinoma
}

\author{
Mitsue Sogawa ${ }^{1}$, Kenji Watanabe ${ }^{1}$, Yutaro Egashira ${ }^{2}$, Kiyoshi Maeda ${ }^{3}$, Kenichi Morimoto $^{1}$, \\ Atsushi Noguchi ${ }^{1}$, Noriko Kamata ${ }^{1}$, Hirokazu Yamagami ${ }^{1}$, Toshio Watanabe ${ }^{1}$, \\ Kazunari Tominaga ${ }^{1}$, Yasuhiro Fujiwara ${ }^{1}$, Nobuhide Oshitani ${ }^{4}$ and Tetsuo Arakawa ${ }^{1}$
}

\begin{abstract}
The patient was a 40-year-old man who had suffered from Crohn's disease (CD) for 19 years and developed an intractable perianal fistula and two strictures in the small bowel. Dilatation of the two strictures using double-balloon endoscopy did not improve the subileus symptoms. An anal canal adenocarcinoma was also detected using double-balloon endoscopy. The ileum and rectoperianal area were partially resected, and a precise immunohistochemical pathologic assessment revealed that all three lesions were fistula-associated adenocarcinomas. Accumulating endoscopic findings of CD-associated cancer and precise pathologic diagnostic findings will help to establish a suitable surveillance method.
\end{abstract}

Key words: Crohn's disease, small bowel adenocarcinoma, double-balloon endoscopy, fistula-associated anal adenocarcinoma, p53

(Intern Med 52: 445-449, 2013)

(DOI: 10.2169/internalmedicine.52.9021)

\section{Introduction}

Several recent studies have highlighted the risk for the development of colorectal carcinoma and small bowel adenocarcinoma (SBA) in patients with Crohn's disease (CD) (1-3). These carcinomas, although relatively rare (4), are associated with poor prognoses due to difficulties in diagnosing them preoperatively. In order to facilitate early diagnosis and establish useful surveillance methods, the clinical, endoscopic and pathologic features of many cases must be evaluated and analyzed. We herein describe the endoscopic and precise pathologic findings of a very rare and remarkable case of a patient with CD having two SBAs and a fistula-associated anal adenocarcinoma (FAAA).

\section{Case Report}

A 40-year-old man with CD complicated by an intractable anal fistula since 1993 was admitted to our hospital due to subileus symptoms in January 2008. He had undergone omental enteroclysis for a small bowel perforation located $180 \mathrm{~cm}$ distal from the ligament of Treitz in October 2005 (Fig. 1). Small bowel enteroclysis showed two strictures (Fig. 2) that were not complicated by any apparent internal fistulae in the proximal or distal ileum. We performed endoscopic balloon dilatation to treat the two strictures with double-balloon endoscopy (DBE) using an oral approach. The first stricture was accompanied by a papillaryprotruding lesion $(220 \mathrm{~cm}$ from the ligament of Treitz; Figs. 1, 3a) and located approximately $40 \mathrm{~cm}$ distal from the

\footnotetext{
${ }^{1}$ Department of Gastroenterology, Osaka City University Graduate School of Medicine, Japan, ${ }^{2}$ Department of Pathology, Osaka Medical College, Japan, ${ }^{3}$ Department of Surgical Oncology, Osaka City University Graduate School of Medicine, Japan and ${ }^{4}$ Department of Gastroenterology, Izumiotsu Municipal Hospital, Japan

Received for publication September 25, 2012; Accepted for publication November 5, 2012

Correspondence to Dr. Mitsue Sogawa, mitsue-s@med.osaka-cu.ac.jp
} 


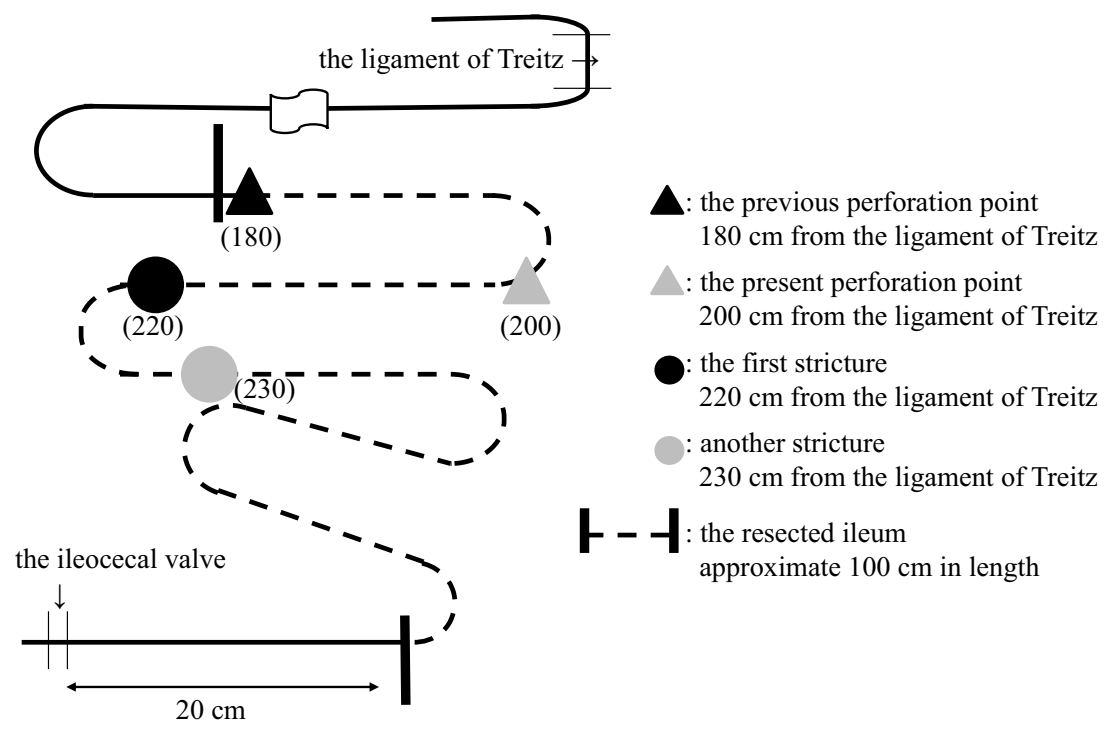

Figure 1. A schematic view of the locations of the two small bowel adenocarcinomas and perforation sites.
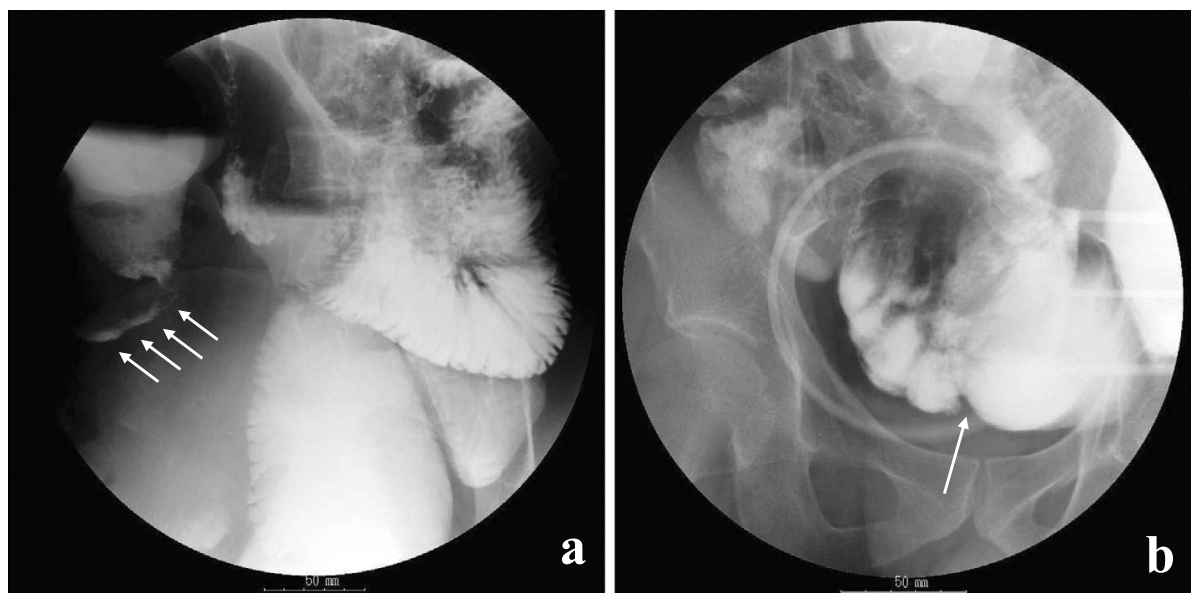

Figure 2. (a) Small bowel enteroclysis showing the stricture that was complicated by a prestenotic dilatation in the proximal ileum. (b) Small bowel enteroclysis showing a stricture in the distal ileum.

previous perforation, while the other stricture was located 10 $\mathrm{cm}$ distal from the first one (Figs. 1, 3b). Unfortunately, the patient's subileus symptoms did not improve despite three successful balloon dilatation procedures. We also performed DBE using an anal approach to observe the other distal lesion before surgery. At that time, we detected a reddish protruding lesion (Fig. 3c) in the anal canal, and the pathologic diagnosis of the biopsy specimen was well-differentiated adenocarcinoma. Therefore, surgical treatment for the ileal strictures and anal canal cancer was scheduled.

Perforation and generalized peritonitis developed suddenly four days before the elective surgery, and the patient underwent emergency partial small bowel resection. The ileum was found to be perforated $200 \mathrm{~cm}$ distal from the ligament of Treitz, $20 \mathrm{~cm}$ proximal to the first stricture (Fig. 1). Approximately $100 \mathrm{~cm}$ of the ileum was resected en bloc from the previously perforated lesion, $20 \mathrm{~cm}$ proximal to the present perforation, to $20 \mathrm{~cm}$ proximal to the ileocecal valve (Fig. 1). The histopathologic diagnosis of the surgical speci- men was a localized well-differentiated adenocarcinoma for the proximal stricture (Figs. 3a, d, 4a, b) and a diffuse and invasive moderately-differentiated adenocarcinoma for the distal stricture (Figs. 3e, 4c-e). A precise pathologic analysis of both lesions revealed that cancer cells lined the inner aspect of the fistula with no marginal dysplasia, and a diffuse Ki-67 expression pattern was observed (the labeling index was $10-50 \%$ for the proximal lesion and $50-70 \%$ for the distal lesion). In addition, p53 was strongly positive immunohistochemically (Fig. 4f, g). The present case was classified as stage T3NOM0 according to the International Union Against Cancer (UICC)/American Joint Committee on Cancer (AJCC) TNM classification system.

After the patient recovered from the emergency surgery, transanal enucleation surgery was attempted. However, because it was difficult to ensure an adequate view, we performed abdominoperineal resection 2.5 months after the emergency surgery (Fig. 3f). No lymph node involvement was detected in the abdomen. The precise pathologic diag- 

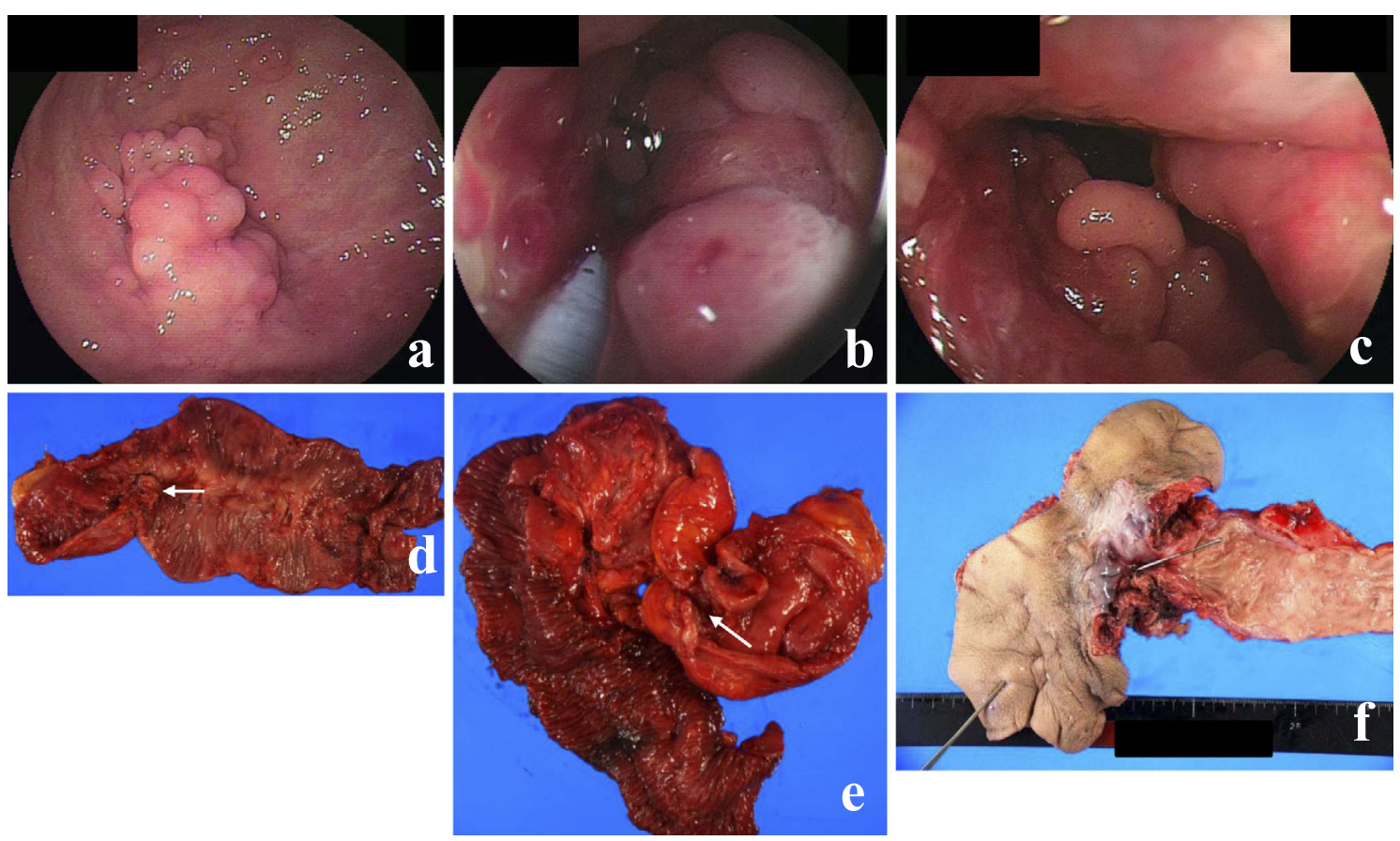

Figure 3. (a) Double-balloon endoscopy (DBE) using an oral approach showing a small bowel stricture $40 \mathrm{~cm}$ distal to the previous perforation $(220 \mathrm{~cm}$ from the ligament of Treitz). This stricture was accompanied by a papillary-protruding lesion. (b) A small bowel stricture $50 \mathrm{~cm}$ distal to the previous perforation ( $230 \mathrm{~cm}$ from the ligament of Treitz) observed on DBE. (c) DBE using an anal approach showing a reddish protruding lesion in the anal canal. The biopsy specimen was pathologically diagnosed as a well-differentiated adenocarcinoma. (d) The macroscopic findings of the resected specimen of the proximal stricture showing that the stricture was accompanied by a papillaryprotruding lesion, similar to the stricture shown in (a). (e) The macroscopic findings of the resected specimen of the distal stricture showing an obstruction due to torsion. (f) The macroscopic findings of the resected rectoperineal specimen showing a perianal fistula.

nosis was a localized mucinous adenocarcinoma (Fig. 5a-c), and cancer cells lined the inner aspect of the fistula with no marginal dysplasia. The Ki-67 expression pattern was diffuse (the labeling index was 10-60\%), and p53 was negative immunohistochemically (Fig. 5d, e). The present case was classified as stage T2N0M0 according to the UICC/AJCC TNM classification system.

We continue to follow this patient, and there has been no evidence of carcinoma recurrence without chemotherapy 42 months after the final surgery.

\section{Discussion}

Chronic inflammation is widely implicated in neoplastic progression, as represented by colorectal carcinomacomplicated inflammatory bowel disease (1). Colonoscopy is the established method of surveillance for ulcerative colitisassociated colorectal carcinoma. In contrast, surveillance methods for CD-associated SBA and perianal fistulaassociated cancer are not yet established because conducting examinations of the deep small bowel insertion is often difficult due to the presence of adhesions. The initial lesions of FAAA in perianal fistulae are usually very difficult to detect using endoscopy, computed tomography or magnetic reso- nance imaging. Despite the significantly higher relative risk of carcinoma in patients with $\mathrm{CD}$, these lesions are rarely diagnosed preoperatively and have a poor prognosis in the early stages (1-3). In a recent study based on a hospital cohort of 1,935 patients with small bowel-involved $\mathrm{CD}$, the cumulative risk of SBA in the patients with CD (95\% confidence interval) was estimated to be $2(0-8)$ and 22 (7-64) per 1,000 patients after 10 and 25 years of follow-up, respectively (3). There have been no population-based studies targeting FAAA; however, the presence of anal carcinomas in patients with $\mathrm{CD}$ is clearly associated with long-standing perianal fistulae $(4,5)$. Twenty-three articles describing 59 patients with adenocarcinoma arising from perianal fistulae in patients with $\mathrm{CD}$ were reviewed by Igors et al. (6). The overall survival rate following abdominoperineal resection was $88 \%$ at one year, $54 \%$ at three years and $26 \%$ at five years (6). Because a significantly increased cancer risk has been described in patients with rectal remnants (7), abdominoperineal resection should be performed early when permanent fecal diversion is suspected.

We herein described the endoscopic and precise pathologic findings of CD-associated SBA. Patients with CD often have inflammatory strictures of the intestines that are usually not considered malignancies. Because DBE is useful 

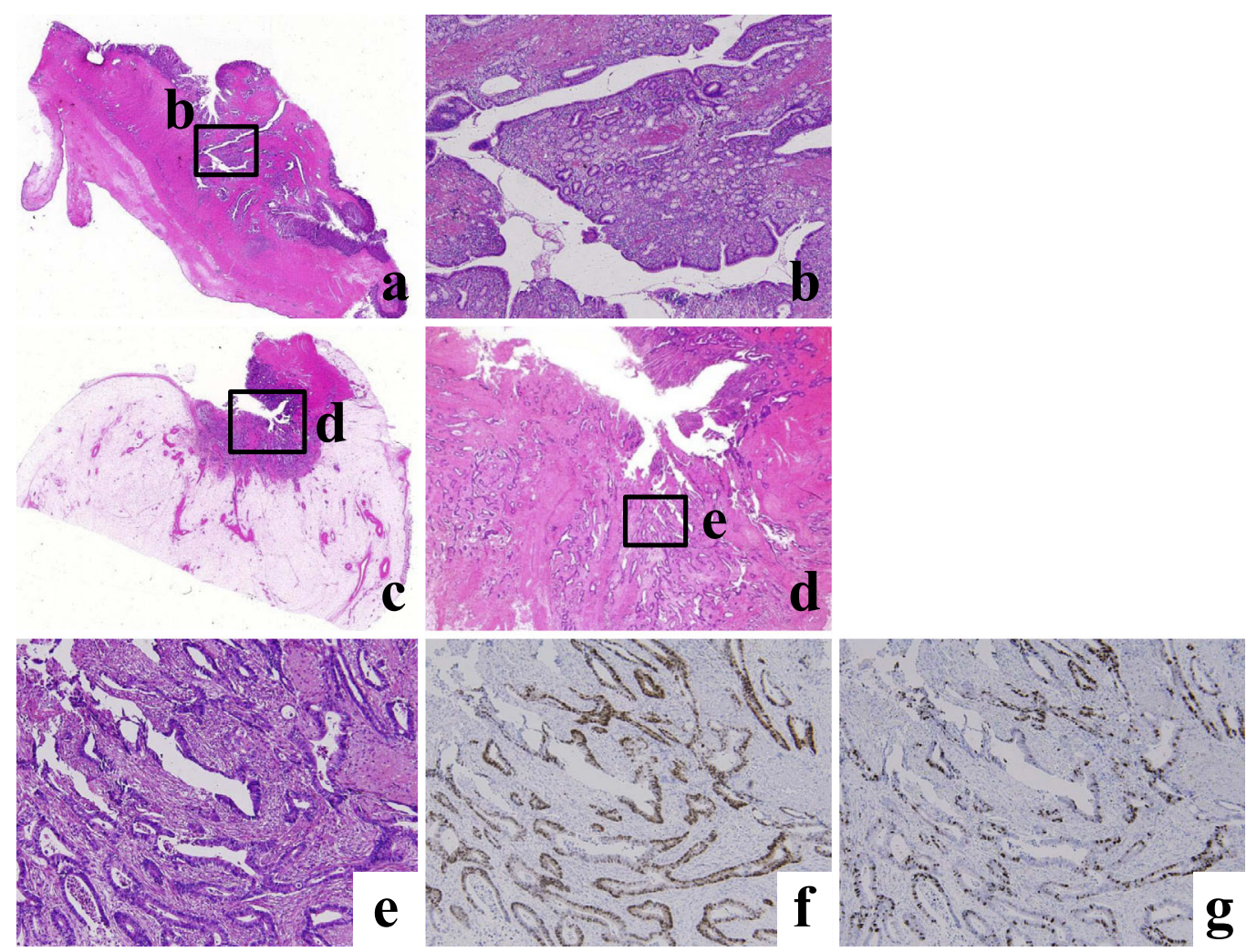

Figure 4. (a) The histologic findings of the resected specimen of the proximal stricture showing a deep fistula and a papillary-protruding lesion. (b) The microscopic findings of the boxed area in (a) showing that cancer cells lined the inner aspect of the fistula. (c) The histologic findings of the resected specimen of the distal stricture showing a transmural fistula. (d) The microscopic findings of the boxed area in (c) showing that cancer cells lined the inner aspect of the fistula. (e) The microscopic findings of the boxed area in (d) showing a well-differentiated adenocarcinoma with no marginal dysplasia. (f) The immunohistological findings of p53 in the same area as shown in (e). The p53 expression was strongly positive. (g) The immunohistological findings of Ki-67 for the same area as shown in (e). The Ki-67 expression exhibited a diffuse pattern.

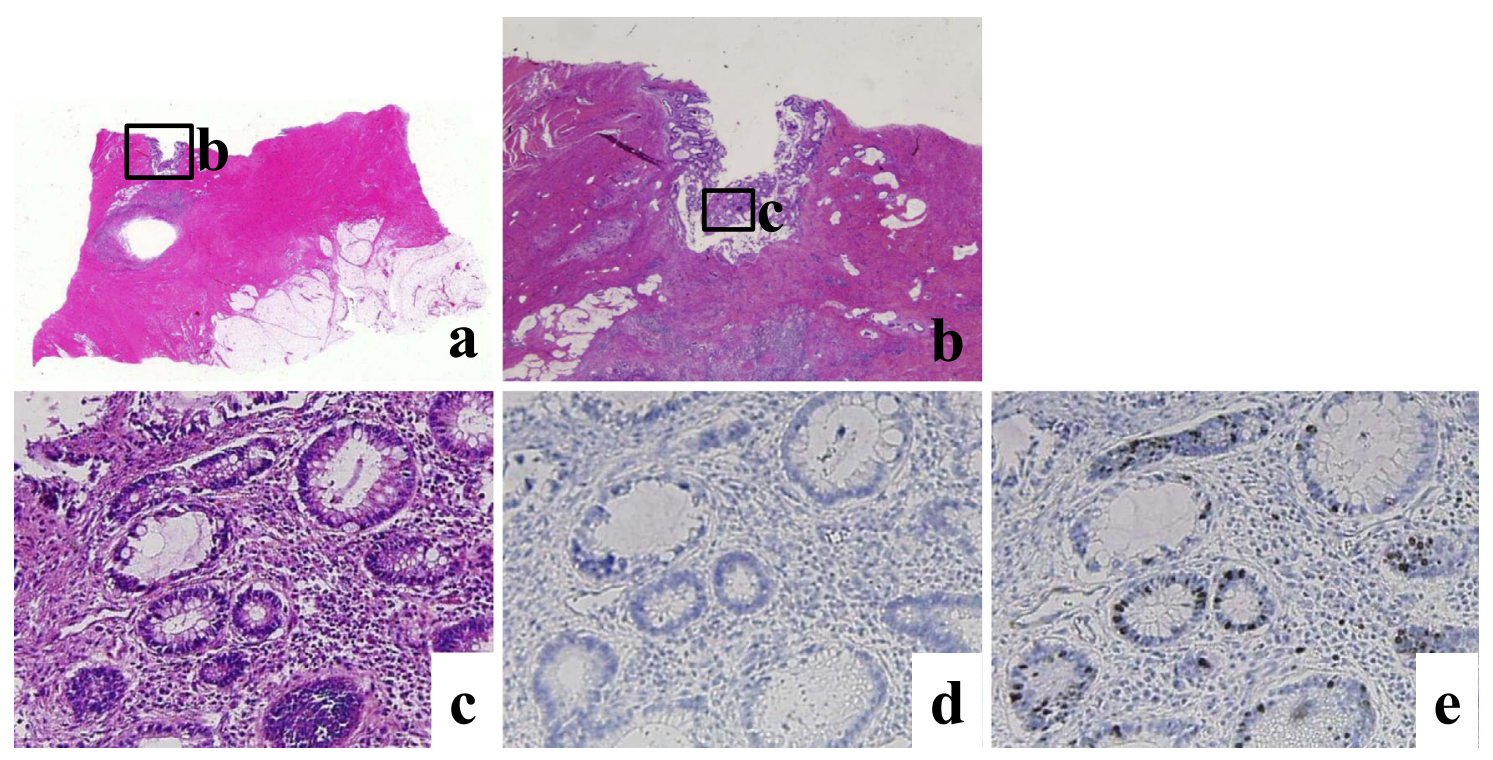

Figure 5. (a) The histologic findings of the resected specimen of the abdominoperineal resection showing a perianal fistula. (b) The microscopic findings of the boxed area in (a) showing that cancer cells lined the inner aspect of the fistula. (c) The microscopic findings of the boxed area in (b). (d) The immunohistological findings of p53 for the same area as shown in (c). The p53 expression was negative. (e) The immunohistological findings of Ki-67 for the same area as shown in (c). The Ki-67 expression exhibited a diffuse pattern. 
for diagnosing SBA $(8,9)$, biopsy specimens should be obtained from small bowel strictures in patients with longstanding $\mathrm{CD}$, especially those refractory to endoscopic balloon dilatation (10). Endoscopic diagnosis of SBA at inflammatory strictures, however, is not yet established in patients with CD. Accumulating endoscopic findings of CDassociated cancer and their precise pathologic diagnostic findings will help to establish suitable surveillance methods. The pathological difference between SBA and FAAA should be evaluated in the near future based on the accumulation of cases including precise pathological diagnoses, as in the present case report. Based on these investigations, early diagnosis of CD-associated cancer, SBA and FAAA, which can be cured by surgery, is an important clinical issue. Furthermore, with the development of anti-tumor necrosis factor agents that provide better prognoses, the establishment of surveillance methods for SBA and FAAA in patients with $\mathrm{CD}$ is important to prevent advanced cancer from developing.

The authors state that they have no Conflict of Interest (COI).

\section{References}

1. Jess T, Loftus EV Jr, Velayos FS, et al. Incidence and prognosis of colorectal dysplasia in inflammatory bowel disease: a population-based study from Olmsted County, Minnesota. Inflamm Bowel Dis 12: 669-676, 2006.

2. Jess T, Gamborg M, Matzen P, et al. Increased risk of intestinal cancer in Crohn's disease: a meta-analysis of population-based cohort studies. Am J Gastroenterol 100: 2724-2749, 2005.

3. Palascak-Juif V, Bouvier AM, Cosnes J, et al. Small bowel adenocarcinoma in patients with Crohn's disease compared with small bowel adenocarcinoma de novo. Inflamm Bowel Dis 11: 828-832, 2005.

4. Barrs JE, Kulpers EJ, Dijkstra G, et al. Malignant transformation of perianal and enterocutaneous fistulas is rare: results of 17 years of follow-up from The Netherlands. Scand J Gastroenterol 46: 319-325, 2011.

5. Ky A, Sohn N, Weinstein MA, et al. Carcinoma arising in anorectal fistulas of Crohn's disease. Dis Colon Rectum 41: 992-996, 1998.

6. Igors I, Wolfgang B, Heidi G, et al. Fistula-associated anal adenocarcinoma in Crohn's disease. Inflamm Bowel Dis 16: 1643-1648, 2010.

7. Sjödahl RI, Myrelid P, Söderholm JD. Anal and rectal cancer in Crohn's disease. Colorectal Dis 5: 490-495, 2003.

8. Yamagami H, Oshitani N, Hosomi S, et al. Usefulness of doubleballoon endoscopy in the diagnosis of malignant small-bowel tumors. Clin Gastroenterol Hepatol 6: 1202-1205, 2008.

9. Kita H, Yamamoto H. Current status of double balloon endoscopy. Dig Endosc 19: 111-115, 2007.

10. Sunada K, Yamamoto H, Kita H, et al. Successful treatment with balloon dilatation using a double-balloon enteroscope for a stricture in the small bowel of a patient with Crohn's disease. Dig Endosc 16: 237-240, 2004.

(C) 2013 The Japanese Society of Internal Medicine http://www.naika.or.jp/imonline/index.html 